\title{
A ATENÇÃO FONOAUdIÓlOGICA E A LINGUAGEM ESCRITA DE PESSOAS COM BAIXA VISÃO: ESTUDO EXPLORATÓRIO
}

SPEECH AND LANGUAge PATHOLOGY THERAPY AND THE READING AND WRITING OF A PERSON

WITH VISUAL DISABILITIE: EXPLORATORY STUDY

\author{
Mayla Myrina Bianchim MONTEIRO ${ }^{1}$ \\ Rita de Cássia letto MONTILHA² \\ Maria Elisabete Rodrigues FreireGASPARETTO³
}

RESU M O: os objetivos deste trabal ho foram: conhecer como as pessoas com baixa visão (visão subnormal) adquirida utilizavam a linguagem escrita no cotidiano erecomendar a atenção fonoaudiológica nesse processo. Foi realizado estudo descritivo exploratório para a construção do instrumento de coleta de dados. A amostra foi constituída por pessoas com baixa visão que freqüentaram o Programa de Reabilitação de A dolescentes e A dultos do Cepre/ FCM/ Unicamp em 2008. A plicou-se questionário por entrevista, onde foram investigadas as variáveis: características pessoais, uso de recursos de tecnologia assistiva na leitura e escrita, razões das atividades de leitura e escrita e frequência do uso após a perda visual. A amostra foi composta por 08 pessoas com baixa visão com média de idade de 47 anos e predominância do sexo masculino (75,0\%). Os resultados indicaram que a maioria $(62,5 \%)$ relatou utilizar auxílios ópticos nas atividades de leitura. Todos informaram utilizar auxílios não ópticos na leitura. Os sujeitos declararam utilizar a leitura para obter informações sobre assuntos que os interessavam ea escrita para se comunicarem com as outras pessoas. Verificou-se quea maioria (75,0\%), relatou não utilizar a leitura e nem a escrita com a mesma freqüência que usava antes da perda visual e os motivos al egados foram a dificuldade para enxergar e o cansaço visual. A redução do uso da linguagem escrita no cotidiano por sujeitos com baixa visão adquirida compromete a autonomia e independência, fato este que demonstra necessidade de ênfase no trabal ho com a linguagem escrita que poderá ser maximizado por meio da atenção fonoaudiológica.

PA LAV RAS-CH AVES: Visão Subnormal. Leitura. Escrita. Fonoaudiologia. Programa de Reabilitação.

ABSTRACT: The aim of this study was to: understand how people with acquired low vision (subnormal vision) used written language in daily living and to recommend speech and language pathology therapy during the process. A descriptive/ exploratory study was conducted in order to build a data collection instrument. The sample was composed of subjects with low vision who attended Cepre / FCM / Unicamp in 2008. A questionnaire was applied during an interview, during which time the following variables were investigated: personal characteristics, use of assistive technology in reading and writing, reasons for performing reading and writing activities and the frequency of reading and writing after having acquired the visual loss. The sample was made up of 8 subjects with acquired low vision. The mean age was 47 years, of which $75,0 \%$ were males. Most of the subjects $(62,5 \%)$ declared they used optical aids to read. All reported they used non-optical aids to read. The results showed that the subjects reported that they used to read to get information on topics

\footnotetext{
1 M estrado pelo Programa de Pós-Graduação Saúde, Interdisciplinaridadee Reabilitação do Centro deEstudos ePesquisas em Reabilitação (CEPRE) da Faculdade deCiências Médicas da UniversidadeEstadual de Campinas (UNICAMP). maylamonteiro@gmail.com

2 Professora Doutora em Ciências Médicas, Área de Concentração-Oftalmologia; Docente da Graduação em Fonoaudiologia e do Centro de Estudos e Pesquisas em Reabilitação (CEPRE) da Faculdade de Ciências Médicas da Universidade Estadual de Campinas (UNICAMP). rcietto@fcm.unicamp.br

3 Professora Doutora em Ciências M édicas. Docente da Graduação em Fonoaudiologia e do Centro de Estudos ePesquisas em Reabilitação (CEPRE) da Faculdade deCiências M édicas da Universidade Estadual deCampinas (UNICAMP). gasparetto@fcm.unicamp.br
} 
of interest and they write to communicate with other people. The majority $(75,0 \%)$ reported they didn't read and write with the same frequency as before the emergence of the ophthalmic problem and the reason given was difficulty in seeing and eyestrain. The reduction of reading and writing for individuals with low vision justifies the need for greater emphasis on working with reading and writing during rehabilitation, and this can be enhanced by speech and language pathology therapy.

KEYWORD S: Special Education. Subnormal Vision. Writing. Speech and Language Pathology. Rehabilitation Program.

\section{InTRODUÇão}

Considerando as atribuições do fonoaudiólogo, o Curso de Graduação em Fonoaudiologia da Unicamp tem sido inovador no oferecimento de atuação interdisciplinar do corpo docentee formação interdisciplinar. Nessecontexto conta com a colaboração de docentes de diversas áreas do conhecimento como terapia ocupacional, serviço social, pedagogia, psicologia, arte educação, linguística, biologia e medicina. Como inovação, também se destaca o oferecimento da disciplina "A Interdisciplinaridade na Reabilitação das Deficiências Sensoriais" queaborda os conteúdos teóricos e práticos referentes à baixa visão, cegueira, surdez e surdocegueira, propiciando ao aluno conhecimentos sobre a deficiência visual, ampliando assim a área de atuação do fonoaudiólogo, o que será de grande diferencial em sua formação abrindo perspectivas no mercado de trabalho.

Como resultado dessa formação, tem se verificado o interesse dos al unos da graduação em uma área pouco explorada pela Área da Fonoaudiol ogia: a deficiência visual. O interesse despertado, a ausência de literatura da deficiência visual dentro da Fonoaudiol ogia e a busca por respostas permearam a realização deste estudo.

Desde cedo, a humanidade sentiu necessidade de registrar, guardar sua linguagem. Foi dessa necessidade que surgiu a escrita. Por causa da própria natureza do signo lingüístico, o homem percebeu desde cedo que havia dois caminhos a seguir (embora, historicamente, um tenha precedido o outro); representar o significado (ideia) veiculado a pal avra e, a partir do reconhecimento destesignificado, chegar aos sons que formam esta palavra, na leitura; representar os sons de uma palavrae, a partir do reconhecimento desses sons, na leitura, chegar ao significado desta palavra, às idéias a ela associada (CA GLIARI, 1993).

A linguagem, segundo Oliveira (2002), éuma das formas de comunicar e expressar sentimentos, ideias, ações, portanto, nossa visão de mundo. É um processo de interlocução que se realiza nas práticas sociais de grupos distintos, em diferentes momentos de sua história. É uma atividadee uma operação mental que implicam a construção de sentidos.

Durante a leitura, a informação extraída da página impressa no que se refere à decodificação e ao reconhecimento da palavra e a apreensão do texto, é analisada ecomparada com as informações previamentearmazenadas. Assim, para 
um entendimento aprofundado do texto, o leitor formula espontaneamente dois tipos de inferências: inferências literais, relacionando idéias dentro ou entre as sentenças e inferências implícitas, conectando idéias para completar informações que não estão explícitas, incorporando conhecimentos e experiências anteriores (SANTOS; NAVAS, 2002).

A linguagem escrita representa uma das grandesáreas da Fonoaudiologia e desta forma, todo os processo de reabilitação que envolve esta área também diz respeito à Fonoaudiologia. O estudo da leitura e da escrita com sujeitos deficientes visuais é um campo novo e pouco explorado pela Fonoaudiologia, mas que tem potencial para contribuir no processo de reabilitação desses sujeitos.

Observa-se que há poucos trabal hos que abrangem a prática do fonoaudiólogo na reabilitação de indivíduos adultos com deficiência visual adquirida. Sabe-se que a Fonoaudiologia trata de um aspecto importante para a socialização do ser humano: a comunicação. Seja esta, por meio da leitura eescrita, ou da fala.

Segundo a Organização Mundial de Saúde (1993), éconsi derado cego o indivíduo com acuidadevisual entre $3 / 60(0,05)$, no mel hor ol ho emel hor correção óptica possível, até ausência de percepção de luz, ou correspondente perda de campo visual no melhor ol ho com a mel hor correção possível. A baixa visão (visão subnormal ) corresponde à acuidade visual igual ou menor do que6/ $18(0,3)$, mas, igual ou maior do que 3/ $60(0,05)$ no melhor ol ho com a melhor correção possível.

A baixa visão caracteriza-se por ser uma alteração significativa da capacidade funcional da visão, decorrente de fatores isolados ou associados, tais como baixa acuidade visual significativa, redução importante do campo visual, alterações para a visão de cor e/ ou sensi bilidade aos contrastes que interferem ou limitam o desempenho visual (BRUNO, 2007).

O estudo de Gasparetto (2001) relata que é importante mencionar que mesmo utilizando a mel hor correção, os indivíduos continuam apresentando baixa visão e o uso de óculos comum nem sempre proporciona melhora quantitativa a essa população, mas, de forma geral, proporciona melhora qualitativa. A mel hora quantitativa propicia ao indivíduo com baixa visão enxergar objetos, símbolos ou letras menores enquanto que a melhora qualitativa propicia a enxergar objetos, símbolos, letras do mesmo tamanho, porém, com maior qualidadee mel hor nitidez.

Salomon (2007) concebe o desenvolvimento da eficiência visual como um processo de aprendizagem em quea pessoa aprenderá a usar seu resíduo visual com os recursos de tecnologia assistiva que poderão beneficiá-lo neste desenvolvimento. Os recursos detecnologia assistiva podem ser ópticos enão ópticos.

Tecnologia assistiva é uma área do conhecimento, de característica interdisciplinar, queengloba produtos, recursos, metodologias, estratégias, práticas e serviços que objetivam promover a funcionalidade, relacionada à atividade e partici pação, de pessoas com deficiência, incapacidades ou mobilidade reduzida, 
visando sua autonomia, independência, qualidade de vida e inclusão social. (GASPARETTO et al., 2009).

Os auxílios não ópticos são recursos simples queaumentam a resolução visual. Podem ser usados em conjunto com o auxílio óptico ou não, com o objetivo demelhorar a função visual. Como exemplos podem ser citados: as lentes filtrantes, iluminação, canetas hidrográficas, materiais ampliados, contraste (fundo escuro e objeto claro) e tiposcópio (guia de leitura) entre outros. Verifica-se que durante o processo de reabilitação os profissionais têm favorecido que as pessoas com baixa visão utilizem tais recursos (KARA-JOSE; TEM PORINI, 1999).

Os auxílios ópticos: os óculos, os sistemas telescópicos, as lupas manuais, de apoio e de mesa, ajudam a melhorar o desempenho visual por meio da magnificação da imagem. De acordo com Burman-Lindelow (2000), a seleção de tais auxílios está baseada nas alterações visuais, nas necessidades visuais e atividades a serem realizadas pelo sujeito que tem baixa visão. A pós a escol ha do auxílio óptico, a adaptação é muito importante. Se a adaptação do auxílio for realizada de forma correta, o auxílio será utilizado corretamente. Caso contrário, certamente ficará numa gaveta e não será usado.

As alternativas de utilização dos recursos de tecnologia assistiva, somadas a real ização de atividades pré-estabel ecidas de acordo com as necessidades individuais, promovem um grande diferencial, levando o sujeito com baixa visão a alcançar o grau máximo de eficiência na utilização da visão residual. Assim, as imagens chegam mais nítidas ao cérebro e conseqüentemente serão melhores interpretadas (ALM EIDA; SILVA, 2008). O uso de recursos detecnologia assistiva (recursos ópticos e não ópticos) na realização das atividades de leitura escrita vai propiciar melhor desempenho visual da pessoa com baixa visão e dessa forma, suprir as suas necessidades.

A ação reabilitadora que pretendemediar a relação do aprendentecom a leitura e a escrita deve incluir atividades que permitam o trabalho com a linguagem em situações reais de uso no cotidiano, incentivando as mais variadas práticas sociais para que tais ações se estendam para além do espaço da sala de atendimento (OLIVEIRA, 2002).

Moya etal. (2002) reconhecendo quea leitura ea escrita são importantes fatores de inclusão social, econômica e cultural, compreende a busca dos sujeitos pela reabilitação no uso das habilidades relacionadas à leitura. Os profissionais deparam-se constantemente com a escassez de materiais para leitura que atendam às necessidades visuais das pessoas com baixa visão e também, se estruture em lingüística: complexidade fonética, morfológica e sintática.

Considerando todos esses aspectos, esta pesquisa tem como objetivos: conhecer como as pessoas com baixa visão adqui rida utilizam a linguagem escrita no cotidiano e recomendar a atenção fonoaudiológica nesse processo. 


\section{Método}

Foi realizado estudo descritivo exploratório para a construção do instrumento decoleta de dados erecebeu aprovação do ComitêdeÉtica em Pesquisa da Faculdade de Ciências Médicas sob o nº91/ 2008.

A amostra foi constituída por 08 pessoas com baixa visão adquirida que foram atendidas no Programa de Reabilitação de Adolescentes e Adultos com Deficiência Visual do Centro de Estudos ePesquisas em Reabilitação Prof. Dr. Gabriel Porto (Cepre), da Faculdade de Ciências Médicas (FCM) da Universidade Estadual de Campinas (Unicamp). Os critérios de inclusão adotados foram: ser adolescente ou adulto com baixa visão adquirida, ser al fabetizado eter participado de um Grupo de Reabilitação do CEPRE no segundo semestre do ano letivo de 2008.

Para a construção do instrumento de col eta de dados (A pêndicel), foi utilizado o estudo exploratório que apresenta natureza qualitativa e contextual (PIOVESA N; TEMPORINI, 1995) e permitequeo pesquisador defina os problemas de sua pesquisa e formule hipóteses de forma mais correta. Permite também que ele escolha o instrumento, mais adequado à sua pesquisa e ajuda a decidir quais questões ele precisa dar maior ênfasee detal har a investigação (TEMPORINI, 1991).

As variáveis selecionadas foram: características pessoais, utilização da linguagem escrita no cotidiano com ou sem auxílios ópticos/ não ópticos, tipos dos auxílios utilizados, finalidade ea freqüência do uso da linguagem escrita após a perda visual.

A primeira fase do estudo exploratório foi realizada por meio de entrevista individual com três pessoas com baixa visão, contendo questões abertas baseadas nas variáveis apresentadas anteriormente. Conjuntamente, também foram realizadas atividades de leitura e escrita com esses sujeitos que foram convidados (individualmente) a lerem com o auxílio óptico e/ ou auxílio não óptico em uso, um pequeno texto impresso que versava sobre uma notícia apresentada na mídia escrita ou falada no período da coleta de dados (agosto a dezembro de 2008), apresentada em diferentes tipos de letras (arial, tahoma e verdana) e tamanhos $(14,16,20,24)$ para que pudessem escolher o que mel hor seadequava à sua situação visual. A pós a leitura, foram realizadas atividades de interpretação, para que o indivídu o fosseestimulado a expor a sua opinião sobre as atividades propostas eo uso da leitura em seu cotidiano.

A pós essas atividades, foram realizadas as de escrita. Foram oferecidas folhas de sulfite A4, cortadas ao meio, com pautas ampliadas e espaçadas; pincéis atômicos para queos sujeitos escrevessem bi lhetes ou texto, relacionado à noticia lida.

A poiando-se nos resultados obtidos nessa etapa, a segunda fase se constituiu na construção de um instrumento semi-estruturado. A s questões foram organizadas de forma a respeitar a ordem dos conteúdos e agrupadas segundo as variáveis do estudo, tendo sido mantida fidelidade à linguagem utilizada pelos sujeitos. Com os dados obtidosna aplicação desta segunda fasefoi possível construir 
o instrumento com questões estruturadas eo questionário foi aplicado a 03 pessoas com baixa visão, que não haviam participado da etapa anterior. Ao encerrar essa fase, verificou-sea necessidade derevisão ecomplementação de al gumas perguntas no questionário, como por exemplo, a freqüência do uso da leitura e escrita após terem adquirido a baixa visão.

A terceira eúltima etapa do estudo exploratório foi compreendida pela reaplicação do questionário a outros dois indivíduos com baixa visão. Não havendo necessidadedealterações, verificou-sequeo instrumento de coleta de dados estava concluído.

\section{Resultados}

A amostra foi composta por oito indivíduos com baixa visão adquirida, cuja participação deu-se da seguinteforma: três indi víduos participaram da $1^{\text {a }}$ fase, três da $2^{\circ}$ fase e dois da $3^{\circ}$ fase. $\mathrm{Na} 1^{\text {a }}$ fase a média de idade foi de 42 anos, na $2^{\text {a }}$ fase de 43 anos e na $3^{\text {a }}$ a média de idade foi de 53 anos. Houve predominância do sexo masculino na $1^{\text {a }}$ fase e na $2^{\text {a }}$ fase, e igualmente na $3^{\text {a }}$ fase.

Dos oito indivíduos com baixa visão participantes, somente cinco responderam o questionário, sendo três da $2^{\text {a }}$ fase do estudo edois da terceira fase. Em relação ao uso de auxílios ópticos na realização das atividades de leitura e escrita, observou-se que dos três indivíduos que participaram da 2 a fasedo estudo, dois $(66,6 \%)$ relataram fazer uso de auxílio óptico e todos os que participaram da $3^{a}$ fase (dois) também mencionaram o uso de auxílios ópticos, sobressaindo-se o uso de óculos e a lupa manual. Esses quatro indivíduos respondentes (80,0\%) declararam utilizar o auxílio óptico para assistir televisão e ler as legendas.

Para auxiliar a leitura, os recursos não ópticos mais citados pelos sujeitos foram,i à aproximação dos textos e objetos aos olhos e o uso de tipos ampliados totalizando $66,6 \%$ na $2^{2}$ fase e $100,0 \%$ na $3^{3}$ fase, conforme apresentados na Tabela 1.

Tabela 1 - Uso de auxílios não ópticos para auxiliar a leitura de pessoas com baixa visão adquirida. Campinas-SP, 2008

\begin{tabular}{lcc}
\hline \multicolumn{1}{c}{ Uso de auxílio não óptico para leitura* } & $\mathrm{n}$ & $\%$ \\
\hline Elaboração do Questionário (2ªse) & & $\mathrm{n}=3$ \\
\hline Aproximação dos objetos aos olhos & 2 & 66,6 \\
Uso de tipos ampliados & 2 & 66,6 \\
Acessibilidade de informática & 1 & 33,3 \\
Melhor qualidade de impressão & 0 & 0 \\
Uso de contraste & 0 & 0 \\
& & $\mathrm{n}=2$ \\
\hline Re-elaboração do Questionário (3 ${ }^{\mathrm{a}}$ fase) & & 100,0 \\
\hline Aproximação dos objetos aos olhos & 2 & 100,0 \\
Uso de tipos ampliados & 2 & 0 \\
Acessibilidade de informática & 0 & 0 \\
Melhor qualidade de impressão & 0 & 0 \\
Uso de contraste & 0 & 0 \\
\hline
\end{tabular}

*Respostas múltiplas 
Tabela 2 - Uso de auxílio não óptico para auxiliar a escrita de pessoas com baixa visão adquirida. Campinas-SP, 2008

\begin{tabular}{lll}
\hline \multicolumn{1}{c}{ Uso de auxílio não óptico para escrita* } & $\mathbf{n}$ & \% \\
\hline Elaboração do Questionário (2 ${ }^{\mathrm{a}}$ fase) & & $\mathrm{n}=3$ \\
\hline Ampliação & 2 & 66,6 \\
Contraste & 1 & 33,3 \\
Maior espaçamento entre as linhas & 0 & 0 \\
& & $\mathrm{n}=2$ \\
\hline Re-elaboração do Questionário (3 ${ }^{a}$ fase) & 2 & 100,0 \\
\hline Ampliação & 0 & 0 \\
Contraste & 0 & 0 \\
Maior espaçamento entre as linhas & & \\
\hline
\end{tabular}

Para auxiliar a escrita, o uso de tipos ampliados e o uso de contrastes foram os recursos mais citados pelos indivíduos (ampliação: $66,6 \%$ na $2^{\text {a }}$ fase e $100,0 \%$ na $3^{a}$ fase; contraste: $33,3 \%$ na $2^{\text {a }}$ fase).

Tabela 3 - Finalidade do uso da leitura de pessoas com baixa visão adquirida. Campinas-SP, 2008

\begin{tabular}{lll}
\hline \multicolumn{1}{c}{ Finalidade do uso da leitura* } & $\mathbf{n}$ & $\%$ \\
\hline Elaboração do Questionário (2 ${ }^{\text {a fase})}$ & & $\mathrm{n}=3$ \\
\hline Para buscar informações que interessavam & 3 & 100,0 \\
Por solicitação de alguma pessoa & 0 & 33,3 \\
Por não ter ninguém para ler por ele & 1 & 33,3 \\
& & $\mathrm{n}=2$ \\
\hline Re-elaboração do Questionário (3 ${ }^{\text {a }}$ fase) & 2 & 100,0 \\
Para buscar informações que interessavam & 1 & 50,0 \\
Por solicitação de alguma pessoa & 0 & 0 \\
Por não ter ninguém para ler por ele & & \\
\hline
\end{tabular}

*Respostas múltiplas

Verificou-se que maioria dos sujeitos (100,0\% na $2^{\mathrm{a}}$ e $3^{\mathrm{a}}$ fase) relatou fazer uso da leitura para buscar informações que eram de seu interesse.

Tabela 4 - Finalidade do uso da escrita por pessoas com baixa visão adquirida. Campinas-SP, 2008.

\begin{tabular}{lll}
\hline \multicolumn{1}{c}{ Finalidade do uso da escrita* } & $\mathbf{n}$ & \% \\
\hline Elaboração do Questionário (2 ${ }^{\text { fase) }}$ & & $\mathrm{n}=3$ \\
\hline Para escrever recados para outras pessoas & 3 & 100,0 \\
Por não ter ninguém que escrevesse por ele & 1 & 33,3 \\
Por solicitação de al guma pessoa & 0 & 0 \\
Para utilização da informática & 1 & 33,3 \\
\hline Re-elaboração do Questionário (3 fase) & & $\mathrm{n}=2$ \\
\hline Para buscar informações que interessavam & 2 & 100,0 \\
Por não ter ninguém que escrevesse por ele & 1 & 50,0 \\
Por solicitação de alguma pessoa & 0 & 0 \\
Para utilização da informática & 1 & 50,0 \\
\hline
\end{tabular}

*Respostas múltiplas 
Em relação à final idadedo uso da escrita, a maioria dos sujeitos (100,0\%) na $2^{\mathrm{a}}$ fase e na $3^{\mathrm{a}}$ fase) relatou utilizá-la para escrever recados para outras pessoas (Tabela 4).

A o serem questionados sobrea utilização da leitura eda escrita após a perda visual, a mai oria dos sujeitos com baixa visão sendo dois da $2^{\text {a }}$ fasee dois da $3^{3}$ fase $(80,0 \%)$, relatou não utilizar a leitura enem a escrita com a mesma freqüência que utilizavam antes do aparecimento do problema oftalmológico, alegando dificuldades visuais para enxergar e cansaço visual.

\section{DısCUSSÃo}

A utilização do estudo exploratório como recurso metodológico permitiu um conhecimento mais completo e mais adequado da realidade, propiciando a construção de um instrumento demedida compatível com a realidade que se pretendia conhecer.

De acordo com Almeida; Silva (2008), os olhos funcionam como uma câmera de altíssima precisão que levam imagens e grafemas ao cérebro para que sejam por eleinterpretados. Porém, esteprocesso sofreal terações devido às doenças oculares que levam à deficiência visual, comprometendo a acuidade visual, o campo visual, percepção de cores, à sensibilidade aos contrastes, ao claro e ao escuro. Estimativas indicam que $80,0 \%$ das pessoas consideradas cegas possuem visão útil, principal mente para o aprendizado da leitura e da escrita.

O equacionamento da deficiência visual écomplexo erequer ações para a promoção da saúde ocular, medidas de prevenção, recursos clínicos com indicação cirúrgica em alguns casos, prescrição de auxílios ópticos/ não ópticos, programas de habilitação e reabilitação realizados por equipe interdisciplinar (SAMPAIO, 2009). Segundo Bruno (2007), a baixa visão éd efinida para fins educacionais como a condição de indicar projeção de luz até o grau em que a redução da acuidade visual interfira ou limita o desempenho visual das pessoas. O processo educativo se desenvolverá principal mente por meios visuais, ainda que com a utilização de recursos específicos.

A sociedade arca com prejuízos elevados em decorrência da falta de atenção com a saúde visual, representados pela diminuição de produtividade da sua força de trabalho e o elevado custa de ações de reabilitação. Acrescem-se a isto, conseqüências psicológicas, sociais eeconômicas para o sujeito com deficiência visual devidas às restrições ocupacionais, diminuição da renda, perda de 'status', de auto-estima, de autoconfiança. (KARA-JOSE; TEM PORINI, 1999).

Por intermédio dos auxílios ópticos e não ópticos, procura-se ampliar a imagem incidente sobre a retina emel horar as condições ambi entais para obter a consequentemelhora da resolução visual (SAMPAIO, 2009). Esses auxílios suprem as necessidades das pessoas com baixa visão nas situações em que os materiais 
impressos estão em tamanho de fonte insuficiente e propiciam a maximização do desempenho visual, autonomia e independência.

O funcionamento visual de um indivíduo portador de baixa visão está relacionado com a maior ou menor capacidade para utilizar o resíduo visual na realização de tarefas cotidianas (GASPARETTO, 2001). Por isso, decorre a necessidade de programas de reabilitação que ensinem eincentivem essas pessoas a utilizarem seus auxílios ópticos de forma a al cançarem melhora quantitativa e qualitativa em seu desempenho visual.

Neste estudo, verificou-se que a maioria das pessoas declarou utilizar auxílios ópticos na real ização das atividades de leitura e escrita no cotidiano (66,6\% na $2^{\text {a }}$ fasee $100,0 \%$ na $3^{a}$ fase). Quando há indicação, a prescrição desses auxílios às pessoas com baixa visão é al tamente necessária pelos motivos apresentados, mas, o que se observa é que nem sempre estão disponíveis, devido ao pequeno número deserviços especial izados queatendem essa população, à situação sócio-econômica que impossi bilita a aquisição dos recursos e a falta de treinamento e orientações para utilização dos mesmos. Em pesquisa sobre o uso de recursos ópticos na atividadedeleitura, Corn; Koenig (2002) verificaram mel hora significativa na leitura deescolares queutilizavam recursosópticos comparadoscom os quenão utilizavam tais recursos.

A nal isand o o conceito deleitura eas complexidades inerentes ao processo, a leitura de textos na avaliação e treinamento de indivíduos com baixa visão, surge como estratégia capaz de determinar quantitativamente (velocidade de leitura) e qual itativamente, a adaptação no uso dos auxílios ópticos (MOYA et al., 2002).

Pesquisa realizada em Campinas por Montilha et al. (2006) mostrou que $85,6 \%$ dos escolares com baixa visão encaminhados pelo Serviço de Visão Subnormal do Hospital de Clínicas da Unicamp tiveram adesão ao uso do auxílio óptico, após freqüentarem o Programa de Reabilitação no Cepre.

Estudo realizado na Inglaterra evidenciou a importância do acompanhamento à adaptação do auxílio óptico. Foram estudados 168 sujeitos com baixa visão que tiveram a prescrição destes auxílios associados ao uso dos auxílios não ópticos. A pós o período de intervenção de seis meses, $88,0 \%$ dos sujeitos apresentaram melhora significativa, estando aptos a lerem letras impressas de jornais (MARGRAIN, 2000).

Sacks (1996) afirmou queo uso deauxílios ópticos podeaumentar a autoestima de sujeitos com baixa visão. Entre os benefícios do uso destes auxílios ressaltam-se: o senso de independência (quando este sujeito consegue ter acesso à leitura de materiais impressos no ambiente diário, ele se torna independente); o senso de responsabilidade (ao adquirir a informação visual real, ele percebe o seu potencial esesenteresponsável eseguro para enxergar em outras situações); mel hora a identificação do ambiente; o senso de competência (porque o sujeito tem controle visual sobre o ambiente) e o sentimento de maior prazer pela qualidade visual. 
A utilização de auxílios ópticos abrange mais do que a melhora da função visual. A brange também o lado emocional do sujeito, tão importante para sua participação efetiva durante o processo de reabilitação, proporcionando melhora na qualidade de vida e inclusão social .

Nesta pesquisa, verificou-se na Tabela 1, que para realização das atividades de leitura os auxílios não ópticos mais apontados pelos sujeitos foram a aproximação dos objetos aos ol hos (66,6\% na $2^{\text {a }}$ fasee $100,0 \%$ na $3^{\text {a }}$ fase) ea utilização de caracteres ampliados (também $66,6 \%$ na $2^{\text {a }}$ fase e $100,0 \%$ na $3^{\text {a }}$ fase). Cabe ressaltar, que a aproximação dos materiais aos ol hos também propicia ampliação do material eéum recurso cujo uso deveser estimulado, mas, nem sempreéusado, por causa do mito de que a aproximação dos olhos aos textos e objetos pode ser prejudicial e acelerar o processo da doença ocular.

Verificou-se que os indivíduos desta pesquisa não indicaram o uso de materiais contrastantes como estratégia para melhorar o desempenho visual em atividades deleitura, apesar dos respondentes terem tido contato com esse recurso por meio dos textos selecionados pelos profissionais que atuam na reabilitação. Estudo de Legge et al. (1992), demonstrou que sujeitos com baixa visão apresentavam melhor desempenho na leitura, quando o material utilizado tinha a impressão negativa (letras brancas em fundo preto), preferência atribuída à dispersão da luz em ol hos com opacidade da córnea e do cristal ino.

Segundo Moya et al. (2002), a leitura é um instrumento adequado nos procedimentos de avaliação e reabilitação, sendo que a leitura de textos subsidia além da avaliação quantitativa, a adaptação dos auxílios por meio do número de palavras lidas corretamente.

A lém disso, a habilidade de leitura tem sido incluída como padrão de medida de visão, etem se mostrado mais sensível na detecção de problemas coma leitura, do quea medida tradicional da acuidadevisual, quando utilizados optotipos isolados (FLETCHER; SCHUCHARD, 2006).

Observou-sena Tabela 2, queem relação ao uso deauxílios não ópticos nas atividades de escrita, destacaram-se o uso de tipos ampliados e do contraste $\left(66,6 \%\right.$ na $2^{\text {a }}$ fase e $100,0 \%$ na $3^{\text {a }}$ fase para tipos ampliados e $33,3 \%$ na $2^{\text {a }}$ fase para uso de contraste). Para a realização das atividades de escrita, é importante que os profissionais verifiquem quais são as necessidades da pessoa com baixa visão, selecionando o mel hor tipo etamanho defonte, considerand o o espaçamento entre as letras e entre as linhas, selecionando também o tipo de pauta (linha) a ser utilizado, considerando o espaçamento, espessura e intensidade da cor. Para melhorar o desempenho da escrita, também pode ser sugerido o uso delápis preto com maior contraste (3B, 4B, 5B ou 6B), canetas hidrográficas e guia de leitura ou o tiposcópio (GASPARETTO, 2010).

De acordo com Cagliari (2001) a escrita para os adultos, é algo tão extremamente envolto no cotidiano que, na maioria das vezes, não se dá conta da 
importância e utilidade à nossa manutenção e sobrevivência. Em um mundo constituído pela palavra escrita, todavia, não se tem o hábito de refletir sobre as dificuldades encontradas por uma pessoa que não domina a escrita e que é diariamente exposta ao conhecimento de ler e escrever.

Verificando-seos resultados da Tabela 3, a maioria das pessoas $(100,0 \%$,

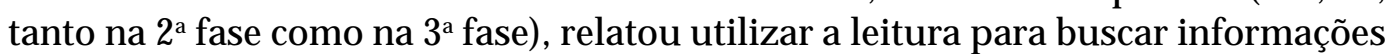
que Ihe interessavam. Os sujeitos com baixa visão que usam a visão como função social, estendem esse uso à realização de atividades cotidianas, às atividades de leitura e escrita, em situações a curta e longa distância e atividades sociais que estão contextualizadas na vida desses sujeitos.

O objetivo dos sujeitos em um processo de comunicação é a busca de entendimento acerca de determinados significados presentes na subjetividade individual além de fazer-se entender pelo interlocutor, levando em consideração o que se espera ser transmitido e o que se espera como resposta (CAMARGO; NARDI, 2008).

A relação entre a função visual e a estratégia de leitura utilizada por sujeitos com baixa visão tem sido intensamente estudada nos últimos anos, e está clara a importância da sua compreensão para a otimização das estratégias de reabilitação (TRAUZETTEL-KLOSIN SKI; HAHN , 2003).

Considerando a complexidadeenvolvida na leitura, Messias et al. (2008) lista que esta deveser avaliada em textos contínuos ecom várias linhas, pois essas são as condições encontradas em jornais, livros, bulas de remédios, etc. Tais textos devem ser padronizados quanto ao tamanho, dificuldade decompreensão esintaxe ea freqüência das palavras para que os resultados obtidos possam ser interpretados corretamente.

Deacordo com Oliveira (2002) ação reabilitadora que pretendemediar a relação do aprendente com a leitura e a escrita deve incluir atividades que permitam o trabalho com a linguagem em situações reais de uso, incentivem as mais variadas práticas sociais, possam ser aplicada a situações do dia-a-dia e permitam o desenvolvimento de projetos que seestendam para além do espaço da sal a de atendimento.

Muitas vezes a pessoa com baixa visão coloca o livro sobre uma mesa tentando ler o texto quase se deitando sobre ele. Tal posição torna-se impossível, porquea pessoa fica cansada eos múscul os do pescoço eombros tornam-setensos. Para realizar as atividades de leitura érecomendad o queessa pessoa sentede forma ereta, movendo a cabeça ou o texto, da forma que se sentir mais confortável. Um simples suporte de livros reduzi rá a fadiga postural por meio da aproximação do material aos olhos da pessoa com baixa visão. Caso o suporte de livros não esteja disponível, pode-se improvisar col ocando outros livros embaixo do livro a ser lido (INDE; BÄCKMAN, 1988).

Na Tabela 4, observou-se que a maioria das pessoas (100,0\%, tanto na 
$2^{\text {a }}$ fase como na $3^{\text {a }}$ fase) relatou utilizar a escrita para redigir recados para outras pessoas. Para A juriaguerra (1988), não se podelimitar a escrita e "seu papel", como fator extremamente motor, pois envolve outras habilidades do ser humano além da cognição, como por exemplo, os aspectos psicomotores que estão envolvidos no processo de traçar formas manuscritas e assim, a capacidade de transmitir a mensagem falada, transformando-as em signos de expressões escritas (não de transcrição da fala, mas respeitando as normas do próprio sistema da escrita estabelecido por cada cultura e tradicionalmente efetivado).

Verificou-se quea maioria das pessoas do estudo (02 indivíduos da $2^{\mathrm{a}}$ fase e 02 da $3^{\text {a }}$ fase, totalizando $80,0 \%$ dos individuos que responderam ao questionário) relatou não utilizar a leitura enem a escrita com a mesma freqüência que usava antes do aparecimento do problema oftalmológico.

O surgimento do problema oftalmológico na idade adulta acarreta mudanças nas atividades de vida diária das pessoas. As tarefas que antes eram feitas com facilidade, após a perda visual, acontecem de manei ra mais difícil, ou, deixam de serem executadas. A leitura e a escrita também passam a adqui rir grau decomplexidademaior, visto que são atividades realizadas essencialmente com a ajuda da função visual e que após a perda, podem apresentar comprometimentos moderados ou graves o que permite compreender as queixas das pessoas dessa pesquisa.

O sistema sensorial, especial mente a visão ea audição fornecem o maior número de receptores ao desenvolvimento da linguagem. As pessoas com baixa visão poderão ter dificuldades na habilidade deintegração destes sistemas de modo a prejudicar a discriminação, a organização, o rastreamento e a memorial visual (ESMERALDO, 2008).

A reaprendizagem ou utilização da leitura e da escrita no processo de reabilitação visual pode vir a ser uma motivação maior da pessoa para usufruí-la com mais prazer e freqüência. Este recurso visaria como resultado uma maior autonomia por parte das pessoas com deficiência visual, auxiliando-as a realizar tarefas cotidianas deleitura eescrita que estavam sendo real izadas por outras pessoas.

Reconhecendo que o Fonoaudiólogo é o profissional que atua na habilitação e reabilitação da comunicação das pessoas, a sua participação nesse processo para as pessoas com baixa visão é de extrema importância, considerando que os sujeitos com déficit visual necessitam utilizar meios diferentes daqueles que estavam acostumados para efetivar a comunicação, possibilitando assim a continuidade de suas relações sociais.

O fonoaudiólogo pode avaliar e intervir com os indivíduos que apresentam baixa visão promovendo associações para o favorecimento de um aprendizado efetivo. Ayres (1989) definiu integração sensorial, como "o processo que organiza as sensações do próprio corpo e do ambiente de forma a ser possível o uso desse corpo no ambiente". 
A atuação fonoaudiológica com os indivíduos com baixa visão, pode utilizar a integração sensorial, por meio da informação de outro sentido, como a linguagem, o tato, o olfato, buscando promover a reciprocidade destes na real ização das atividades cotidianas. A fonoaudiologia tem papel fundamental no desenvolvimento da comunicação do indivíduo e no seu processo de inclusão na sociedade, já quea linguagem éuma habilidade humana que promove as relações sociais (ESM ERA LDO, 2008).

\section{Con CLUSÃo}

Os participantes do estudo relataram utilizar a leitura, principalmente para obter informações sobre assuntos que os interessavam. A maior parte dos sujeitos declarou fazer uso da escrita para escrever bilhetes para outras pessoas, ou seja, utilizavam a escrita para se comunicarem com as outras pessoas que estavam ao seu redor, utilizando-a como um recurso a mais na comunicação.

Verificou-se que as pessoas com baixa visão relataram não utilizar a leitura enem a escrita com a mesma freqüência que usavam antes do aparecimento do problema oftalmológico, alegando dificuldades visuais para enxergar as letras e cansaço visual. A redução do uso da leitura e da escrita por sujeitos com baixa visão, justifica a necessi dade de maior ênfase no trabal ho com a leitura e a escrita durante o processo de reabilitação.

A reaprendizagem da leitura e escrita no processo de reabilitação propicia maior motivação à pessoa com baixa visão, para usufruí-la com maior qualidade de vida efreqüência. Esse recurso real izado por equipe interdisciplinar visa mai or autonomia por parte do indivíduo com deficiência visual, auxiliando-o a realizar as atividades de leitura e escrita no cotidiano.

\section{Referências}

AJURIA GUERRA, J. A . Escrita infantil: evolução edificuldades. Porto Alegre: A rtes Médicas. 1988.

ALMEIDA, J.M.S.; SILVA, C.G. The visual function of people with low vision and their implications in the development of reading. In: SIMPÓSIO NACIONAL DE LETRASE LINGUÍSTICA, 11., SIMPÓSIO INTERNA CIONAL DE LETRA SE LINGUÍSTICA, 1., 2008. Uberlândia. A nais... Uberlândia: UFU. Disponível em: http:/ / www.mel.ileel.ufu.br/ silel2006/ inicial.asp. A cesso em: 13 nov. 2008.

AYRES, A.J. Sensory integration and práxis tests. Los A ngeles: Western Psychological Services, 1989.

BRUNO, M.M.G. A inclusão da criança com baixa visão na Educação Infantil. In: MASINI, E.F.S.; GA SPA RETTO, M .E.R.F. V isão Subnormal : um enfoque educacional. São Paulo: Vetor, p.81-92, 2007. 
BURMAN-LINDELOW, P. Magnificação eauxíliosópticos em baixa visão. In:VEITZMAN, S. (Org.). V isão subnormal. Rio de Janeiro: Cultura Médica, 2000, p.111-122.

CAGLIARI, C.L. A Ifabetização \& lingüística. 10. ed. São Paulo: Sapione, 2001.

. Escrita ideográfica \& escrita fonográfica. Jornal da A Ifabetização. Porto A legre: Kuarup, n. 28, p.18-20, 1993.

CAMARGO, P.C.; NARDI, R. O emprego de linguagens acessíveis para alunos com deficiência visual em aulas de óptica. Revista Brasileira de Educação Especial, Marília, v.14, n.3, p.149-168, 2008.

COLEMBRANDER, A. Reabilitação da baixa visão. In: VEITZMAN, S. (Org.) Visão subnormal. Rio de Janeiro: Cultura Médica, 2000, p. 87-110.

CORN , A.L.; KOEN IG , A.J. Literacy for students with low vision: a framework for delivering instruction. Jounal of V isual Impairment and Blindness. Tennesse, v. 96, p. 305-321, 2002.

ESMERA LDO, L.R. A bordagem fonoaudiológica em crianças com baixa visão por catarata. In: VERÇOSA, I.C.; TARTA RELLA, M.R. (Org.). Catarata na criança. Fortaleza: Celigráfica, 2008, p.304-305.

FLETCHER, D.C.; SCHUCHARD R.A. Visual function in patientes with choroidal neovascularation resulting from age-related macular degeneration: theimportant of looking beyond visual acuity. 0 ptometry \& V ision Science. v. 83, n.3, p.178-189, 2006.

GASPA RETTO, M.E.R.F. Orientações ao professor e à comunidade escolar referentes ao aluno com baixa visão. In: SAMPAIO, M.W. et al. (Org). Baixa visão e cegue ira: os caminhos para a reabilitação, a educação e a inclusão. Rio de Janeiro: Cultura Médica, Guanabara Koogan, 2010, p.347-360.

GASPARETTO, M.E.R.F. et al. Uso de recursos e equipamentos de tecnologia assistiva na educação municipal, estadual e federal tecnológica. In: BRA SIL. Subsecretaria nacional de promoção dos direitos da pessoa com deficiência. Comitê de ajudas técnicas. (Org.). Tecnologia assistiva, Brasília: Corde, p. 41-58, 2009.

. V isão subnormal em escol as públicas: conhecimento, opinião e conduta de professores e diretores do ensino fundamental. 2001. 197 f. Tese (Doutorado em Ciências Médicas). Faculdade de Ciências Médicas, Universidade Estadual de Campinas, Campinas, 2001.

INDE, K.; BÄCKMAN, O. El adiestramiento de la visión subnormal. Madrid: Grefol, 1988.

KARA-JOSE, N.; TEMPORINI, E.R. Cirurgia de catarata: o porquê dos excluídos. Revista Panamericana de Salud Publica, Washington, v.6, n.4, p.242-248, 1999.

LEGGE, G.E. et al. Phychophysics of reading. Clinical predictors of low-vision reading speed. Investigative O phthamololy and V isual Science, Minneapolis, v.33, n.3, p.677-687, 1992.

MARGRAIN , T.H. Helping blind and partially sighted people to read: the effectiveness of low vision aids. British Journal of O phthalmology, Cardiff, v.84, n.8, p.919-921, 2000.

MESSIAS, A. et al. Textos padronizados em português (BR) para medida da vel ocidade de leitura - comparação com quatro idiomas europeus. A rq Bras 0 ftal mol, v.71, n.4, p.553-558, 2008. 
MONTILHA, R.C.I. et al. Utilização de recursos ópticos e equipamentos por escolares com deficiência visual. A rq Bras 0 ftalmol, v.69, n.2, p.207-211, 2006.

MOYA, S.T.F. et al. N ova proposta de treinamento eavaliação do uso deauxílios ópticos em portadores de visão subnormal. A rq Bras 0 ftalmol, v.65, n.1, p.43-47, 2002.

NILSSON, U.L. Visual rehabilitation with and without educational training in the use of optical aids and residual vision. A prospectivestudy of patients with advanced age related macular degeneration. Clinical V isual Science, New York. v. 6, p.3-10, 1990.

OLIVEIRA, L.L. Uma possi bilidade de intervenção psicopedagógica no processo dialético de mediação entre o sujei to que aprende e o objeto do conhecimento. In: SA NTOS, MTM.; NAVAS, A.L.G.P. (Org.). D istúrbios de leitura e escrita: teoria e prática. São Paulo: Manole, 2002, p.169-190.

PIOVENSAN, A.; TEMPORINI, E.R. Pesquisa exploratória: procedimento metodológico para o estudo defatores humanos no campo da saúde pública. R ev. Saúde Pública. v.29, n.4, p.318-325, 1995.

SACKS, S. Z. Psychological and social implications of low vision. In: CORN , A. L.; KOEN IG, A. J. Foundation of low vision: clinical and functional perspective. New York: American Foundation for The Blind Press, 1996, p.26-42.

SALOMON, S.M. Desenvolvimento da eficiência visual: a relação eos atendimentos à pessoa com visão subnormal. In: MASINI, E.F.S.; GASPA RETTO, M.E.R.F. (Org.) V isãoSubnormal: um enfoque educacional. São Paulo: Vetor, 2007, p.95-99.

SAMPAIO, M.W.S. Estimulo ao uso da visão residual. In: KARA-JOSÉ, N.; RODRIGUES, M.L.V. Saúde ocular e prevenção da cegueira. Rio de Janeiro: Cultura M édica, 2009, p. 160-162.

SANTOS, M.T.M.; NAVAS A.L.G.P. Terapia de linguagem escrita. In: SANTOS, M.T.M.; NAVAS, A.L.G.P. D istúrbios de leitura e escrita: teoria e prática. São Paulo: Manole, 2002, p.191-224.

TEMPORINI, E.R. Pesquisa de oftal mologia em saúde pública: considerações metodológicas sobre fatores humanos. A rq. B ras. O ftalmol, v.54, n.6, p.279-281, 1991.

TRA UZETTEL-KLOSINSKI, S.; HAHN, G.A. Support for patients loosing sight. D evelopments in O phthalmology, v.37, p.199-214, 2003.

Recebido: 29/ 07/ 2010

Reformulado: 15/ 03/ 2011

A provado: 04/ 04/ 2011 
APÊNDICE I - Instrumento de coleta de dados

A LEITURA E A ESCRITA DA PESSOA COM BAIXA VISÃO ADQUIRIDA

1. Sexo: Masculino ( ) Feminino ( )

2. Quantos anos completos o(a) sr(a) tem?

3. O(a) sr(a) utiliza algum 'óculos especial' (recurso óptico)? Se sim, qual?

( ) Não

( ) Sim

4. Para quais atividades o(a) sr(a) utiliza o 'óculos especial' (recurso óptico)? Cite exemplos.

5. O(a) sr(a) utiliza algum ‘material’ (recursos não-ópticos) para realizar atividades?

( ) Não

( ) Sim

( ) Régua para leitura (Tiposcópio)

( ) Letras maiores (A mpliação de letras)

( ) Fundo claro e objeto escuro (Contraste)

( ) Iluminação adequada

( ) Suporte para leitura e escrita

( ) Aproximar

( ) Outros

6. Para quais atividades o(a) sr(a) utiliza o 'material' (recurso não-óptico)? Cite exemplos

7. $\mathrm{O}(\mathrm{a}) \mathrm{sr}(\mathrm{a})$ faz uso da leitura:

( ) Para buscar informações que me interessam. O quê?

( ) Quando alguém pede para eu ler

( ) Quando não tem ninguém mais para ler pra mim

( ) Utilizo a leitura sempre

( ) Não faço uso de leitura

8. O(a) sr(a) usa al guns desses recursos para facilitar a sua leitura?

( ) Não

( ) Sim

( ) Melhor qualidade de impressão

( ) Letras maiores (A mpliação de letras)

( ) Fundo claro e objeto escuro (Contraste)

( ) Ler no computador

( ) Colocar mais perto do olho

( ) Outros

9. $\mathrm{O}(\mathrm{a}) \operatorname{sr}(\mathrm{a})$ utiliza a leitura com a mesma freqüência que usava antes do aparecimento do seu problema visual?

( ) Não Por quê?

( ) Sim. Por quê?

10. Para que o(a) sr(a) faz uso da escrita:

( ) Para escrever recados para outras pessoas

( ) Quando não há ninguém para escrever por mim

( ) Quando alguém pede para eu escrever

( ) Quando uso o computador

( ) Anotações para mim mesmo(a)

( ) Não faço uso da escrita

11. $\mathrm{O}(\mathrm{a}) \mathrm{sr}(\mathrm{a})$ usa alguns desses recursos para facilitar a sua escrita?

( ) Linhas mais grossas

( ) Maior espaço entre as linhas

( ) Canetas hidrográficas

( ) Fundo claro e objeto escuro (Contraste)

( ) Escrever no computador

( ) Escrever com letras grandes

( ) Outros

12. O(a) sr(a) utiliza outros meios além da escrita para obter informações que lhe interessam? Se sim, quais? ( ) Não ( ) Sim. Quais?

13. O(a) sr(a) utiliza a escrita com a mesma freqüência que usava antes do aparecimento do seu problema visual?

( ) Não Por quê?

( ) Sim. Por quê? 